\title{
Article \\ Effect of Sleep Bruxism Intensity on Blood Pressure in Normotensives
}

\author{
Monika Michalek-Zrabkowska ${ }^{1}$ (D), Mieszko Wieckiewicz ${ }^{2}$, Pawel Gac $^{3}$ (D), Joanna Smardz ${ }^{2}, \operatorname{Rafal~Poreba~}^{1}$, \\ Anna Wojakowska ${ }^{1}$, Katarzyna Goslawska ${ }^{1}$, Grzegorz Mazur ${ }^{1}$ and Helena Martynowicz ${ }^{1, *}$
}

1 Department of Internal Medicine, Occupational Diseases, Hypertension and Clinical Oncology, Wroclaw Medical University, 213 Borowska St., 50-556 Wroclaw, Poland; monika.michalek@student.umed.wroc.pl (M.M.-Z.); rafal.poreba@umed.wroc.pl (R.P.); anna.wojakowska@umed.wroc.pl (A.W.); k.goslawska@hotmail.com (K.G.); grzegorz.mazur@umed.wroc.pl (G.M.)

2 Department of Experimental Dentistry, Wroclaw Medical University, 26 Krakowska St., 50-425 Wroclaw, Poland; mieszko.wieckiewicz@umed.wroc.pl (M.W.); joanna.smardz@umed.wroc.pl (J.S.)

3 Department of Hygiene, Wroclaw Medical University, 7 Mikulicza-Radeckiego St., 50-345 Wroclaw, Poland; pawel.gac@umed.wroc.pl

* Correspondence: helena.martynowicz@umed.wroc.pl; Tel.: +48-71-736-40-52

\section{check for} updates

Citation: Michalek-Zrabkowska, M.; Wieckiewicz, M.; Gac, P.; Smardz, J.; Poreba, R.; Wojakowska, A.; Goslawska, K.; Mazur, G.; Martynowicz, H. Effect of Sleep Bruxism Intensity on Blood Pressure in Normotensives. J. Clin. Med. 2021, 10, 1304. https://doi.org/10.3390/ jcm10061304

Academic Editor: Giovanni Cammaroto

Received: 22 February 2021

Accepted: 17 March 2021

Published: 22 March 2021

Publisher's Note: MDPI stays neutral with regard to jurisdictional claims in published maps and institutional affiliations.

Copyright: (c) 2021 by the authors. Licensee MDPI, Basel, Switzerland. This article is an open access article distributed under the terms and conditions of the Creative Commons Attribution (CC BY) license (https:// creativecommons.org/licenses/by/ $4.0 /)$.

\begin{abstract}
The present research aimed to investigate the influence of sleep bruxism (SB) intensity on blood pressure parameters in normotensive subjects by using an ambulatory blood pressure device. The study group included 65 normotensive individuals suspected of having SB. All participants underwent one-night video-polysomnography, and ambulatory blood pressure monitoring was performed the next day; $86.15 \%$ of them were diagnosed with SB. Statistical analysis included correlation and regression analysis. The obtained results showed that systolic blood pressure variability during sleep significantly increased in individuals with BEI > 4 (bruxism episodes index; episodes/hour) compared to those with $\mathrm{BEI} \leq 4(8.81 \pm 3.36$ versus $10.57 \pm 3.39, p=0.05)$. Multivariable regression analysis showed that systolic blood pressure variability at nighttime was also associated with the following independent risk factors: higher apnea-to-bruxism index, male gender, BEI $>4$ episodes/hour, body mass index (BMI) $\geq 25 \mathrm{~kg} / \mathrm{m}^{2}$, higher arousal index, and shorter total sleep time. In summary, sleep bruxism intensity was associated with increased systolic blood pressure variability during sleep. Coincidental apnea, male gender, severe sleep bruxism (SB intensity with BEI $>4$ /hour), excess weight and obesity, higher arousal index, and shorter sleep time seem to be the main determinants that influence blood pressure in normotensive sleep bruxers.
\end{abstract}

Keywords: sleep bruxism; ambulatory blood pressure monitoring; polysomnography

\section{Introduction}

Nocturnal drop in blood pressure (BP) is a physiological component of circadian rhythm. [1] The dipping of BP at nighttime is an effect of sympathetic tone decrease during sleep. Absent or diminished decrease in BP during sleep is associated with increased cardiovascular mortality and morbidity [2,3]. Arterial hypertension (HTA) has been reported to be linked with a large number of sleep disturbances: short sleep duration [4], insomnia [5], restless leg syndrome [6], and obstructive sleep apnea (OSA) [7].

Sleep bruxism (SB) is a common sleep-related disorder. The etiology of SB is multifactorial and is still insufficiently explored. Several theories on sleep bruxism etiology and pathophysiology have been proposed; some of these theories focus on a genetic basis and neurotransmission [8], while others focus on psychological risk factors or sympathetic hyperreactivity. The prevalence of SB in adults is estimated between $3 \%$ and $8 \%[9,10]$. Clinical manifestations of SB include tooth grinding and/or jaw clenching, abnormal tooth wear, and jaw muscle pain due to the fact of jaw muscle activity (rhythmic masticatory mus- 
cle activity (RMMA)) during sleep and are classified in the 3rd edition of the International Classification of Sleep Disorders [11].

As shown in the literature, SB commonly coexists with obstructive sleep apnea (OSA) [12]. A recent systematic review by Van Ryswyk et al. [12] examined a large number of epidemiological studies on the relationship between sleep-related breathing disorders and hypertension. As explored in a cross-sectional, multicenter Sleep Heart Health Study (SHHS), the odds ratio increased and was estimated to be 1.37 (95\% confidence interval (CI), 1.03-1.83) for hypertension for subjects with severe OSA compared to that in individuals without OSA [13]. The relationship between OSA and HTA was also confirmed by the Wisconsin Study [14] and the Zaragoza Sleep Cohort Study [15]. The pathomechanism linking increased mortality in apneic patients with HTA refers to BP variability (BPV), with a gradual rise in $\mathrm{BP}$ at the beginning of an apneic/hypopneic event and then abrupt fall in BP after the respiratory event [16]. Recurrent episodes of hypoxic events cause increased cardiovascular morbidity and mortality due to the oxidative stress [17].

Previous studies have emphasized the role of increased sympathetic activity preceding the SB event [18]. A repetitive surge in sympathetic activity expressed as increased heart rate followed by an increase in electroencephalography (EEG) frequency (microarousal) precedes RMMA [19]. Correspondingly, an autonomic sympathetic activation is a key component of pathophysiological repercussions of HTA, for example, vascular, cardiac, renal, metabolic, and immune-related abnormalities [20].

As previously reported in the literature, HTA is correlated with sleep bruxism. A seminal contribution has been made by Nashed et al. [21], who revealed that BP fluctuations during sleep were associated with RMMA. In another study by Martynowicz et al. [22], bruxism intensity was found to be increased in hypertensives. It is worth noting that the design of this study was focused on the assessment of intensity (frequency) and prevalence of SB in patients with HTA compared to that in non-hypertensive subjects.

To date, only a few studies have investigated the association of SB with hypertension. To fill this gap in the literature, the current descriptive study aimed to assess the relationship between ambulatory blood pressure measurements and SB intensity in individuals without HTA.

\section{Materials and Methods}

Sixty-five subjects suspected of having SB were admitted to the Department of Internal Medicine, Occupational Diseases, Hypertension, and Clinical Oncology at the Wroclaw Medical University in Poland. Before admission, all participants underwent complex dental examination at the Clinic of Prosthetic Dentistry at Wroclaw Medical University. The dental examination involved physical examination (focusing on clinical indicators of bruxism [23]) and a medical interview. Individuals who met the criteria for the presence of probable SB according to an international consensus on the assessment of bruxism were included in the study group [24].

The inclusion criteria for the study were as follows: age above 18 years, diagnosis of probable SB based on dental examination, and willingness to participate in the study. The exclusion criteria were diagnosis of HTA or use of anti-hypertensive medications; use of medicines affecting the nervous and muscular systems; cognitive disabilities and inability to provide informed consent; inability to undergo polysomnography (PSG) or measurement with an ambulatory blood pressure monitoring (ABPM) device; severe physical and mental conditions; presence of diabetes, coronary artery disease, active malignancy, or inflammation.

All participants signed a consent form and voluntarily underwent examination. The study was approved by the local Ethics Committee (no. KB-195/2017) and was conducted by adopting the principles of the Declaration of Helsinki. This clinical trial was also registered in Clinical Trials Database (www.ClinicalTrials.gov, accessed on: 20 March 2021): identifier NCT03083405, WMU1/2017. 
Polysomnographic examinations were conducted in the sleep laboratory at the Department of Internal Medicine, Occupational Diseases, Hypertension, and Clinical Oncology at the Wroclaw Medical University in Poland. The PSG data were collected using a Nox-A1 (Nox Medical, Reykjavík, Iceland) device for one-night video-PSG. Recordings included sleep, bruxism, and respiratory data as follows: sleep latency, total sleep time, and sleep efficiency (\%); the ratio of N1 (sleep stage 1), N2 (sleep stage 2), N3 (sleep stage 3), and the stage of REM (rapid eye-movement sleep); electromyographic (EMG) recordings of bilateral masseter muscle activity during sleep; video and audio recordings; respiratory events recorded by a nasal pressure transducer; and arterial oxygen saturation $\left(\mathrm{SpO}_{2}\right)$ measured by a finger pulse oximeter.

After conducting an automatic analysis, $30 \mathrm{~s}$ epochs of polysomnograms were evaluated by a certified polysomnographist according to the gold standard criteria of the American Academy of Sleep Medicine (AASM) task force [25]. Respiratory events were scored as follows: the absence of airflow for $\geq 10 \mathrm{~s}$ was scored as an apneic event, while a reduction in the amplitude of breathing by $\geq 30 \%$ for $\geq 10 \mathrm{~s}$ with $\mathrm{a} \geq 3 \%$ decline in blood oxygen saturation or arousal was scored as hypopnea. Sleep bruxism was assessed audiovisually from EMG measurements supplemented by audio and video recordings. The increase in EMG amplitude that was at least twice that of the background EMG was considered as a bruxism episode. Electromyographic bursts within $3 \mathrm{~s}$ were considered to be the part of the same episode. The bruxism episode index (BEI) was determined as the number of bruxism events per hour of sleep, and the SB intensity was classified as insignificant $(\mathrm{BEI}<2)$, mild or moderate (BEI $=2-4)$, or severe (BEI $>4$ ) [23]. The apnea-to-bruxism index refers to a bruxism event secondary to a respiratory event. Temporal association between SB and respiratory events were demonstrated and evaluated previously, suggesting that SB events were secondary to apneic-hypopneic events [26]. As there are no reference values, the authors assumed an apnea-to-bruxism index of $<1$ as normal, concluding that an apnea-to-bruxism index greater than 1 may suggest other etiology of bruxism events, e.g., primary bruxism or against-hypoxia protective features of SB.

The study group consisted of normotensive individuals. Normotension was defined as the lack of use of any antihypertensive medications in medical history and was based on the office BP measurements. According to European guidelines for the management of HTA, individuals with BP values of at least $140 / 90 \mathrm{mmHg}$ were considered as normotensive [27]. All patients included in the study had an office BP measurement in the normal range at the day of admission to internal ward. On the first night, individuals underwent full-night video-polysomnography. Blood pressure measurements were conducted the next day. After conducting PSG, in the morning, patients received ABPM for $24 \mathrm{~h}$ home use as a mandatory requirement for the diagnosis of hypertension. The ABPM devices were attached by a qualified technician, and the measurement was performed according to recommendations of European Society of Hypertension [28]. First, the technician measured the circumference of the upper arm and selected the appropriate cuff. Next, all participants were instructed about the correct position during BP measurement, and the technicians read through the instruction paper together with the patient before $24 \mathrm{~h}$ measurements and explained the possible hazards in detail on the basis of the warnings included in the instructions. The ABPMs were conducted with validated Spacelabs OnTrak Ambulatory Blood Pressure Monitor appliances. The measurement intervals estimated were 15 and 20 min during daytime and nighttime periods, respectively. Daytime was recorded as the period between the time of attachment of the device by the patient and the time when the patient went to bed. Nighttime was registered as the period between the time when the patient went to bed and the time when the patient woke up in the morning. Subsequently, the devices were collected from patients on the following day. The ABPM measurements were scored according to the cut off values in the ESH/ESC (the European Society of Hypertension and the European Society of Cardiology) guidelines for 2018 [27] and extrapolated with findings from PSG. 
Statistical analyses were performed using Dell Statistica 13.1 statistical package (Dell Inc., Round Rock, TX, USA). The distribution of variables was assessed with the W-ShapiroWilk test. For independent quantitative variables with normal distribution, the $t$-test was used for further statistical analysis. For variables with non-normal distribution, the Mann-Whitney U test was used. Correlation and regression analyses were performed to determine the relationship between the studied variables. The model parameters obtained in the regression analysis were estimated using the least squares method. The results at the level of $p<0.05$ were considered to be statistically significant.

\section{Results}

The study group consisted of 65 normotensive individuals; the mean age of the participants was $33.96 \pm 11.22$ years, and $20(30.77 \%)$ were men and $45(69.23 \%)$ were women. The average body mass estimated was $65.12 \pm 13.22 \mathrm{~kg}$, the mean height was $1.70 \pm 0.08 \mathrm{~m}$, and the average body mass index (BMI) (calculated as weight in kilogram divided by square of height in meters) was $22.48 \pm 3.87 \mathrm{~kg} / \mathrm{m}^{2}$. According to BMI, three subjects were identified to be underweight $\left(\mathrm{BMI}<18.5 \mathrm{~kg} / \mathrm{m}^{2}\right), 33 \mathrm{had}$ normal weight $\left(B M I=18.5-25 \mathrm{~kg} / \mathrm{m}^{2}\right)$, nine were overweight $\left(B M I=25-30 \mathrm{~kg} / \mathrm{m}^{2}\right)$, and three were obese $\left(\right.$ BMI $\left.>30 \mathrm{~kg} / \mathrm{m}^{2}\right)$.

After one-night PSG, $56(86.15 \%)$ participants met the criteria for SB, of which 25 were diagnosed with mild $/$ moderate SB (BEI $=2-4$ events $/ \mathrm{h}$ ) and 31 had severe SB (BEI $>4$ events $/ \mathrm{h}$ ). On the basis of the apnea-hypopnea index (AHI), 14 (21.54\%) participants were diagnosed to have OSA, including eight $(12.31 \%)$ with mild OSA (AHI $=5-15$ events $/ \mathrm{h})$, three $(4.62 \%)$ with moderate OSA (AHI $=15-30$ events $/ \mathrm{h}$ ), and three $(4.2 \%)$ with severe OSA (AHI > 30 events $/ \mathrm{h}$ ).

None of the patients in the study group met the criteria for accompanying diseases such as hypertension, diabetes mellitus, or coronary artery disease. The polysomnographic and ABPM parameters in the studied group are presented in Tables 1 and 2.

Table 1. Polysomnographic characteristics of the study group.

\begin{tabular}{lcc}
\hline \multicolumn{1}{c}{ Parameter } & Mean & SD \\
\hline AHI $(n / \mathrm{h})$ & 5.07 & 8.62 \\
ODI $(n / \mathrm{h})$ & 4.76 & 7.48 \\
Snore $(\%$ of TST) & 7.35 & 14.74 \\
TST (min) & 428.00 & 65.50 \\
SL (min) & 22.76 & 21.91 \\
REML (min) & 100.03 & 62.31 \\
WASO (min) & 31.12 & 31.19 \\
SE $(\%)$ & 86.30 & 10.60 \\
N1 $(\%$ of TST) & 4.48 & 3.75 \\
N2 $\%$ of TST) & 49.92 & 7.59 \\
N3 (\% of TST) & 22.65 & 7.02 \\
REM $(\%$ of TST) & 22.95 & 5.26 \\
Arousals (n/h) & 5.04 & 3.92 \\
Mean SpO $(\%)$ & 94.65 & 1.80 \\
Min SpO $(\%)$ & 88.62 & 6.16 \\
SpO $<90 \%(\%)$ & 1.48 & 4.74 \\
Mean desaturation drop $(\%)$ & 3.23 & 0.60 \\
Mean heart rate (beats/min) & 60.67 & 6.84 \\
Max heart rate (beats/min) & 95.97 & 11.79 \\
Min heart rate (beats/min) & 48.52 & 5.98 \\
BEI $(n / h)$ & 5.06 & 3.54 \\
Phasic $(n / h)$ & 3.86 & 7.27 \\
Tonic $(n / h)$ & 2.34 & 2.29 \\
Mixed $(n / h)$ & 1.30 & 1.59 \\
Apnea-to-bruxism index & 1.09 & 1.99
\end{tabular}

AHI, apnea-hypopnea index; ODI, oxygen desaturation index; TST, total sleep time (min); SL, sleep latency; REML, REM latency; WASO, wake after sleep onset; SE, sleep efficiency; N1, sleep stage 1; N2, sleep stage 2; N3, sleep stage 3; REM, rapid eye-movement sleep stage; mean $\mathrm{SpO}_{2}$, mean oxygen saturation (\%); $\mathrm{SpO}_{2}<90 \%$, time with oxygen saturation $<90 \%$ (\% of TST); BEI, bruxism episode index. 
Table 2. Ambulatory BP characteristics of the study group (values are shown as mean \pm SD).

\begin{tabular}{|c|c|c|c|c|}
\hline & Variable & 24 h Mean & Daytime & Nighttime \\
\hline \multirow{5}{*}{ SBP } & Average (mmHg) & $110.56 \pm 7.91$ & $114.03 \pm 8.78$ & $101.68 \pm 7.94$ \\
\hline & Variability $(\mathrm{mmHg})$ & $12.61 \pm 2.99$ & $11.09 \pm 2.68$ & $9.66 \pm 3.46$ \\
\hline & Minimum (mmHg) & $84.75 \pm 7.43$ & $90.59 \pm 9.46$ & $85.55 \pm 8.05$ \\
\hline & Maximum (mmHg) & $141.88 \pm 14.10$ & $141.67 \pm 14.07$ & $121.75 \pm 12.92$ \\
\hline & Decline $(\%)$ & $10.67 \pm 6.06$ & & \\
\hline \multirow{5}{*}{ DBP } & Average (mmHg) & $68.83 \pm 6.08$ & $72.31 \pm 7.12$ & $60.60 \pm 6.55$ \\
\hline & Variability (mmHg) & $11.19 \pm 2.65$ & $9.98 \pm 2.89$ & $8.48 \pm 3.00$ \\
\hline & Minimum (mmHg) & $46.81 \pm 6.58$ & $51.73 \pm 8.08$ & $47.15 \pm 6.42$ \\
\hline & Maximum (mmHg) & $99.64 \pm 15.29$ & $99.09 \pm 14.91$ & $78.25 \pm 12.26$ \\
\hline & Decline $(\%)$ & $15.75 \pm 7.24$ & & \\
\hline \multirow{5}{*}{ MAP } & Average (mmHg) & $83.16 \pm 6.11$ & $86.13 \pm 6.82$ & $75.37 \pm 6.37$ \\
\hline & Variability (mmHg) & $10.79 \pm 2.42$ & $9.74 \pm 2.53$ & $8.07 \pm 2.99$ \\
\hline & Minimum (mmHg) & $61.56 \pm 6.49$ & $65.61 \pm 7.47$ & $62.17 \pm 6.63$ \\
\hline & Maximum (mmHg) & $113.28 \pm 14.07$ & $112.39 \pm 13.87$ & $91.97 \pm 12.33$ \\
\hline & Decline (\%) & $12.49 \pm 6.00$ & & \\
\hline \multirow{4}{*}{$\mathrm{PP}$} & Average (mmHg) & $41.77 \pm 4.97$ & $42.02 \pm 5.21$ & $41.03 \pm 5.03$ \\
\hline & Variability (mmHg) & $7.24 \pm 1.95$ & $7.45 \pm 2.07$ & $5.88 \pm 1.81$ \\
\hline & Minimum (mmHg) & $23.80 \pm 3.23$ & $24.47 \pm 4.16$ & $29.35 \pm 5.13$ \\
\hline & Maximum $(\mathrm{mmHg})$ & $61.86 \pm 9.97$ & $61.00 \pm 10.01$ & $53.62 \pm 8.31$ \\
\hline \multirow{4}{*}{ HR } & Average (beats/min) & $72.09 \pm 6.60$ & $75.31 \pm 7.24$ & $63.35 \pm 6.35$ \\
\hline & $\begin{array}{l}\text { Variability } \\
\text { (beats/min) }\end{array}$ & $12.82 \pm 3.27$ & $12.42 \pm 3.69$ & $7.20 \pm 3.46$ \\
\hline & $\begin{array}{l}\text { Minimum } \\
\text { (beats/min) }\end{array}$ & $52.88 \pm 5.98$ & $55.39 \pm 7.30$ & $54.07 \pm 5.32$ \\
\hline & $\begin{array}{l}\text { Maximum } \\
\text { (beats } / \mathrm{min} \text { ) }\end{array}$ & $112.55 \pm 20.49$ & $111.77 \pm 20.10$ & $83.62 \pm 15.87$ \\
\hline
\end{tabular}

$\overline{\mathrm{SBP}}$, systolic blood pressure; DBP, diastolic blood pressure; MAP, mean arterial pressure; PP, pulse pressure; HR, heart rate.

The difference in the ambulatory BP data among participants with BEI $<2$ and those with BEI $>2$ was nonsignificant, but the group of non-bruxers was relatively small $(n=9)$. However, significant differences in ABPM findings were observed between severe bruxers and the remaining study group (Table 3 ).

Table 3. Ambulatory BP characteristics of patients with and without severe bruxism (BEI $>4$ per hour).

\begin{tabular}{|c|c|c|c|c|c|}
\hline & & ible & $\mathrm{BEI} \leq 4$ & BEI $>4$ & $p$ \\
\hline \multirow{13}{*}{$\mathrm{SBP}(\mathrm{mmHg})$} & \multirow{5}{*}{$24 \mathrm{~h}$ mean } & Average & $111.55 \pm 8.42$ & $109.52 \pm 7.31$ & 0.31 \\
\hline & & Variability & $12.59 \pm 3.27$ & $12.62 \pm 2.71$ & 0.97 \\
\hline & & Minimum & $86.39 \pm 7.39$ & $83.00 \pm 7.19$ & 0.07 \\
\hline & & Maximum & $143.97 \pm 15.21$ & $139.65 \pm 12.67$ & 0.22 \\
\hline & & Decline $(\%)$ & $10.87 \pm 7.15$ & $10.46 \pm 4.75$ & 0.80 \\
\hline & \multirow{4}{*}{ Daytime } & Average & $115.18 \pm 9.54$ & $112.81 \pm 7.85$ & 0.28 \\
\hline & & Variability & $10.86 \pm 2.79$ & $11.32 \pm 2.58$ & 0.50 \\
\hline & & Minimum & $92.21 \pm 10.59$ & $88.87 \pm 8.04$ & 0.16 \\
\hline & & Maximum & $143.97 \pm 15.21$ & $139.23 \pm 12.52$ & 0.18 \\
\hline & \multirow{4}{*}{ Nighttime } & Average & $102.45 \pm 7.98$ & $100.86 \pm 7.95$ & 0.44 \\
\hline & & Variability & $8.81 \pm 3.36$ & $10.57 \pm 3.39$ & 0.05 \\
\hline & & Minimum & $88.13 \pm 8.43$ & $82.79 \pm 6.72$ & 0.01 \\
\hline & & Maximum & $121.10 \pm 13.04$ & $122.45 \pm 12.98$ & 0.69 \\
\hline
\end{tabular}


Table 3. Cont.

\begin{tabular}{|c|c|c|c|c|c|}
\hline & & able & $\mathrm{BEI} \leq 4$ & BEI $>4$ & $p$ \\
\hline \multirow{13}{*}{ DBP (mmHg) } & \multirow{5}{*}{$24 \mathrm{~h}$ mean } & Average & $68.97 \pm 6.44$ & $68.68 \pm 5.78$ & 0.85 \\
\hline & & Variability & $11.21 \pm 2.76$ & $11.17 \pm 2.56$ & 0.95 \\
\hline & & Minimum & $47.91 \pm 6.78$ & $45.65 \pm 6.25$ & 0.17 \\
\hline & & Maximum & $102.24 \pm 15.98$ & $96.87 \pm 14.26$ & 0.16 \\
\hline & & Decline (\%) & $15.79 \pm 8.88$ & $15.71 \pm 5.11$ & 0.96 \\
\hline & \multirow{4}{*}{ Daytime } & Average & $72.09 \pm 7.29$ & $72.55 \pm 7.04$ & 0.80 \\
\hline & & Variability & $10.06 \pm 3.01$ & $9.91 \pm 2.79$ & 0.84 \\
\hline & & Minimum & $52.48 \pm 8.19$ & $50.94 \pm 8.02$ & 0.45 \\
\hline & & Maximum & $102.21 \pm 16.00$ & $95.77 \pm 13.09$ & 0.08 \\
\hline & \multirow{4}{*}{ Nighttime } & Average & $60.77 \pm 7.13$ & $60.41 \pm 5.99$ & 0.83 \\
\hline & & Variability & $7.99 \pm 3.13$ & $9.00 \pm 2.82$ & 0.20 \\
\hline & & Minimum & $48.52 \pm 6.99$ & $45.69 \pm 5.50$ & 0.09 \\
\hline & & Maximum & $77.71 \pm 11.89$ & $78.83 \pm 12.83$ & 0.73 \\
\hline \multirow{13}{*}{ MAP (mmHg) } & \multirow{5}{*}{$24 \mathrm{~h}$ mean } & Average & $83.64 \pm 6.35$ & $82.65 \pm 5.90$ & 0.52 \\
\hline & & Variability & $10.86 \pm 2.53$ & $10.71 \pm 2.34$ & 0.80 \\
\hline & & Minimum & $62.33 \pm 6.87$ & $60.74 \pm 6.07$ & 0.33 \\
\hline & & Maximum & $116.09 \pm 15.04$ & $110.29 \pm 12.50$ & 0.10 \\
\hline & & Decline (\%) & $12.62 \pm 7.24$ & $12.35 \pm 4.45$ & 0.87 \\
\hline & \multirow{4}{*}{ Daytime } & Average & $86.58 \pm 7.37$ & $85.65 \pm 6.26$ & 0.59 \\
\hline & & Variability & $9.83 \pm 2.61$ & $9.64 \pm 2.49$ & 0.76 \\
\hline & & Minimum & $66.24 \pm 8.46$ & $64.94 \pm 6.32$ & 0.49 \\
\hline & & Maximum & $115.79 \pm 15.04$ & $108.77 \pm 11.68$ & 0.04 \\
\hline & \multirow{4}{*}{ Nighttime } & Average & $75.81 \pm 6.71$ & $74.90 \pm 6.07$ & 0.58 \\
\hline & & Variability & $7.58 \pm 2.92$ & $8.59 \pm 3.03$ & 0.20 \\
\hline & & Minimum & $63.74 \pm 7.16$ & $60.48 \pm 5.66$ & 0.06 \\
\hline & & Maximum & $91.48 \pm 12.28$ & $92.48 \pm 12.57$ & 0.76 \\
\hline \multirow{12}{*}{ PP (mmHg) } & \multirow{4}{*}{$24 \mathrm{~h}$ mean } & Average & $42.73 \pm 5.37$ & $40.74 \pm 4.35$ & 0.11 \\
\hline & & Variability & $7.36 \pm 2.08$ & $7.12 \pm 1.82$ & 0.63 \\
\hline & & Minimum & $24.00 \pm 2.85$ & $23.58 \pm 3.62$ & 0.61 \\
\hline & & Maximum & $62.97 \pm 10.35$ & $60.68 \pm 9.57$ & 0.36 \\
\hline & \multirow{4}{*}{ Daytime } & Average & $43.06 \pm 5.65$ & $40.90 \pm 4.52$ & 0.10 \\
\hline & & Variability & $7.61 \pm 2.26$ & $7.28 \pm 1.87$ & 0.53 \\
\hline & & Minimum & $24.73 \pm 4.31$ & $24.19 \pm 4.05$ & 0.61 \\
\hline & & Maximum & $62.52 \pm 10.64$ & $59.39 \pm 9.19$ & 0.21 \\
\hline & \multirow{4}{*}{ Nighttime } & Average & $41.65 \pm 5.24$ & $40.38 \pm 4.81$ & 0.33 \\
\hline & & Variability & $5.58 \pm 1.72$ & $6.20 \pm 1.89$ & 0.19 \\
\hline & & Minimum & $30.48 \pm 5.39$ & $28.14 \pm 4.63$ & 0.08 \\
\hline & & Maximum & $52.87 \pm 7.83$ & $54.41 \pm 8.87$ & 0.48 \\
\hline \multirow{12}{*}{$\begin{array}{c}\text { HR } \\
\text { (beats/min) }\end{array}$} & \multirow{4}{*}{$24 \mathrm{~h}$ mean } & Average & $72.24 \pm 6.56$ & $71.94 \pm 6.76$ & 0.85 \\
\hline & & Variability & $13.64 \pm 3.67$ & $11.95 \pm 2.57$ & 0.04 \\
\hline & & Minimum & $53.24 \pm 6.17$ & $52.48 \pm 5.85$ & 0.62 \\
\hline & & Maximum & $117.82 \pm 23.15$ & $106.94 \pm 15.70$ & 0.03 \\
\hline & \multirow{4}{*}{ Daytime } & Average & $75.45 \pm 6.96$ & $75.16 \pm 7.65$ & 0.87 \\
\hline & & Variability & $13.33 \pm 4.25$ & $11.45 \pm 2.72$ & 0.04 \\
\hline & & Minimum & $55.82 \pm 7.68$ & $54.94 \pm 6.98$ & 0.63 \\
\hline & & Maximum & $116.76 \pm 22.42$ & $106.45 \pm 15.99$ & 0.04 \\
\hline & \multirow{4}{*}{ Nighttime } & Average & $64.03 \pm 7.17$ & $62.62 \pm 5.36$ & 0.39 \\
\hline & & Variability & $7.65 \pm 4.18$ & $6.71 \pm 2.44$ & 0.30 \\
\hline & & Minimum & $54.77 \pm 5.46$ & $53.31 \pm 5.15$ & 0.29 \\
\hline & & Maximum & $87.84 \pm 18.84$ & $79.10 \pm 10.46$ & 0.03 \\
\hline
\end{tabular}

$\overline{\mathrm{SBP}}$, systolic blood pressure; DBP, diastolic blood pressure; MAP, mean arterial pressure; PP, pulse pressure; HR, heart rate; values are shown as mean $\pm \mathrm{SD}$, statistically significant differences marked as bold $(p<0.05)$. 
Differences in the ambulatory BP data between individuals with an apnea-to-bruxism index $<1$ and those with an apnea-to-bruxism index $\geq 1$ was statistically significant (Table 4).

Table 4. Comparison of ambulatory BP data among study groups with apnea-to-bruxism indexes $<1$ and apnea-to-bruxism indexes $\geq 1$.

\begin{tabular}{|c|c|c|c|c|c|}
\hline & & ible & Apnea-to-Bruxism $<1$ & Apnea-to-Bruxism $\geq 1$ & $p$ \\
\hline \multirow{13}{*}{ SBP (mmHg) } & \multirow{5}{*}{$24 \mathrm{~h}$ mean } & Average & $109.28 \pm 7.16$ & $113.83 \pm 8.95$ & 0.04 \\
\hline & & Variability & $12.13 \pm 2.92$ & $13.82 \pm 2.91$ & 0.04 \\
\hline & & Minimum & $84.96 \pm 7.54$ & $84.22 \pm 7.34$ & 0.73 \\
\hline & & Maximum & $139.74 \pm 12.92$ & $147.33 \pm 15.83$ & 0.05 \\
\hline & & Decline $(\%)$ & $10.45 \pm 6.39$ & $11.22 \pm 5.26$ & 0.66 \\
\hline & \multirow{4}{*}{ Daytime } & Average & $112.57 \pm 7.97$ & $117.78 \pm 9.84$ & 0.03 \\
\hline & & Variability & $10.74 \pm 2.36$ & $11.98 \pm 3.28$ & 0.10 \\
\hline & & Minimum & $89.87 \pm 9.11$ & $92.44 \pm 10.36$ & 0.33 \\
\hline & & Maximum & $139.74 \pm 12.92$ & $146.61 \pm 15.99$ & 0.08 \\
\hline & \multirow{4}{*}{ Nighttime } & Average & $100.53 \pm 7.54$ & $104.59 \pm 8.41$ & 0.07 \\
\hline & & Variability & $8.88 \pm 3.24$ & $11.64 \pm 3.30$ & 0.00 \\
\hline & & Minimum & $86.02 \pm 8.38$ & $84.35 \pm 7.25$ & 0.47 \\
\hline & & Maximum & $119.37 \pm 10.80$ & $127.76 \pm 15.97$ & 0.02 \\
\hline \multirow{13}{*}{ DBP (mmHg) } & \multirow{5}{*}{$24 \mathrm{~h}$ mean } & Average & $67.93 \pm 5.81$ & $71.11 \pm 6.34$ & 0.06 \\
\hline & & Variability & $10.94 \pm 2.50$ & $11.82 \pm 2.97$ & 0.24 \\
\hline & & Minimum & $47.20 \pm 6.97$ & $45.83 \pm 5.51$ & 0.46 \\
\hline & & Maximum & $98.22 \pm 13.87$ & $103.28 \pm 18.39$ & 0.24 \\
\hline & & Decline (\%) & $15.78 \pm 7.97$ & $15.69 \pm 5.16$ & 0.97 \\
\hline & \multirow{4}{*}{ Daytime } & Average & $70.96 \pm 6.40$ & $75.78 \pm 7.68$ & 0.01 \\
\hline & & Variability & $9.94 \pm 2.56$ & $10.10 \pm 3.68$ & 0.84 \\
\hline & & Minimum & $51.04 \pm 7.49$ & $53.50 \pm 9.44$ & 0.28 \\
\hline & & Maximum & $98.13 \pm 13.97$ & $101.56 \pm 17.25$ & 0.41 \\
\hline & \multirow{4}{*}{ Nighttime } & Average & $59.70 \pm 6.64$ & $62.88 \pm 5.89$ & 0.09 \\
\hline & & Variability & $7.92 \pm 2.85$ & $9.87 \pm 3.00$ & 0.02 \\
\hline & & Minimum & $47.53 \pm 6.81$ & $46.18 \pm 5.39$ & 0.47 \\
\hline & & Maximum & $75.98 \pm 8.69$ & $84.00 \pm 17.54$ & 0.02 \\
\hline \multirow{13}{*}{ MAP (mmHg) } & \multirow{5}{*}{$24 \mathrm{~h}$ mean } & Average & $82.15 \pm 5.63$ & $85.72 \pm 6.69$ & 0.03 \\
\hline & & Variability & $10.52 \pm 2.33$ & $11.48 \pm 2.58$ & 0.16 \\
\hline & & Minimum & $61.50 \pm 6.59$ & $61.72 \pm 6.42$ & 0.90 \\
\hline & & Maximum & $111.83 \pm 13.01$ & $117.00 \pm 16.27$ & 0.19 \\
\hline & & Decline (\%) & $12.49 \pm 6.48$ & $12.49 \pm 4.75$ & 1.00 \\
\hline & \multirow{4}{*}{ Daytime } & Average & $84.98 \pm 6.29$ & $89.06 \pm 7.39$ & 0.03 \\
\hline & & Variability & $9.62 \pm 2.21$ & $10.02 \pm 3.27$ & 0.57 \\
\hline & & Minimum & $64.74 \pm 6.89$ & $67.83 \pm 8.60$ & 0.14 \\
\hline & & Maximum & $111.61 \pm 12.94$ & $114.39 \pm 16.24$ & 0.48 \\
\hline & \multirow{4}{*}{ Nighttime } & Average & $74.40 \pm 6.09$ & $77.82 \pm 6.58$ & 0.06 \\
\hline & & Variability & $7.48 \pm 2.82$ & $9.57 \pm 2.97$ & 0.01 \\
\hline & & Minimum & $62.26 \pm 6.81$ & $61.94 \pm 6.35$ & 0.87 \\
\hline & & Maximum & $89.58 \pm 9.60$ & $98.00 \pm 16.26$ & 0.02 \\
\hline
\end{tabular}


Table 4. Cont.

\begin{tabular}{|c|c|c|c|c|c|}
\hline & & able & Apnea-to-Bruxism $<1$ & Apnea-to-Bruxism $\geq 1$ & $p$ \\
\hline \multirow{12}{*}{$\mathrm{PP}(\mathrm{mmHg})$} & \multirow{4}{*}{$24 \mathrm{~h}$ mean } & Average & $41.41 \pm 4.97$ & $42.67 \pm 4.97$ & 0.37 \\
\hline & & Variability & $7.02 \pm 1.83$ & $7.82 \pm 2.17$ & 0.14 \\
\hline & & Minimum & $23.65 \pm 2.62$ & $24.17 \pm 4.50$ & 0.57 \\
\hline & & Maximum & $61.28 \pm 9.94$ & $63.33 \pm 10.17$ & 0.46 \\
\hline & \multirow{4}{*}{ Daytime } & Average & $41.61 \pm 5.14$ & $43.06 \pm 5.40$ & 0.32 \\
\hline & & Variability & $7.25 \pm 1.98$ & $7.95 \pm 2.26$ & 0.23 \\
\hline & & Minimum & $24.17 \pm 3.84$ & $25.22 \pm 4.93$ & 0.37 \\
\hline & & Maximum & $60.52 \pm 10.08$ & $62.22 \pm 10.01$ & 0.55 \\
\hline & \multirow{4}{*}{ Nighttime } & Average & $40.86 \pm 5.04$ & $41.47 \pm 5.15$ & 0.68 \\
\hline & & Variability & $5.50 \pm 1.52$ & $6.84 \pm 2.16$ & 0.01 \\
\hline & & Minimum & $29.86 \pm 4.95$ & $28.06 \pm 5.51$ & 0.22 \\
\hline & & Maximum & $52.16 \pm 7.92$ & $57.29 \pm 8.39$ & 0.03 \\
\hline \multirow{12}{*}{ HR (beats/min) } & \multirow{4}{*}{$24 \mathrm{~h}$ mean } & Average & $72.74 \pm 5.63$ & $70.44 \pm 8.58$ & 0.21 \\
\hline & & Variability & $13.39 \pm 3.04$ & $11.34 \pm 3.48$ & 0.02 \\
\hline & & Minimum & $53.30 \pm 5.70$ & $51.78 \pm 6.68$ & 0.36 \\
\hline & & Maximum & $116.00 \pm 20.41$ & $103.72 \pm 18.40$ & 0.03 \\
\hline & \multirow{4}{*}{ Daytime } & Average & $75.89 \pm 6.06$ & $73.83 \pm 9.69$ & 0.31 \\
\hline & & Variability & $13.06 \pm 3.47$ & $10.77 \pm 3.82$ & 0.02 \\
\hline & & Minimum & $56.02 \pm 6.60$ & $53.78 \pm 8.85$ & 0.27 \\
\hline & & Maximum & $115.24 \pm 19.75$ & $102.89 \pm 18.67$ & 0.03 \\
\hline & \multirow{4}{*}{ Nighttime } & Average & $64.14 \pm 6.16$ & $61.35 \pm 6.56$ & 0.13 \\
\hline & & Variability & $7.67 \pm 3.68$ & $5.99 \pm 2.53$ & 0.09 \\
\hline & & Minimum & $54.44 \pm 5.29$ & $53.12 \pm 5.43$ & 0.39 \\
\hline & & Maximum & $86.56 \pm 16.42$ & $76.18 \pm 11.78$ & 0.02 \\
\hline
\end{tabular}

SBP, systolic blood pressure; DBP, diastolic blood pressure; MAP, mean arterial pressure; PP, pulse pressure; HR, heart rate; values are shown as mean $\pm \mathrm{SD}$, statistically significant differences marked as bold $(p<0.05)$.

Regression analysis was performed to determine the factors independently associated with mean arterial pressure (MAP) (average from the $24 \mathrm{~h}$ mean of APBM and daytime measurements) and systolic blood pressure (SBP) variability during sleep period. In the regression model, we used all potentially independent variables: apnea-to-bruxism index, male gender, $\mathrm{BMI}$, age, $\mathrm{BEI}>4, \mathrm{BMI} \geq 25$, arousal index, and total sleep time (TST).

The regression model showed that a higher apnea-to-bruxism index and higher BMI were independently associated with the increased average of $24 \mathrm{~h}$ mean of MAP (Table 5).

Table 5. Results of estimation for the final model obtained on multivariate regression analysis. model for average MAP (24 h Mean).

\begin{tabular}{lccc}
\hline \multirow{2}{*}{ Parameter } & \multicolumn{3}{c}{ Model for Average MAP (24 h Mean) } \\
\cline { 2 - 4 } & Rc & SEM of RC & p \\
\hline Intercept & $\mathbf{6 8 . 3 1}$ & $\mathbf{4 . 7 2}$ & $\mathbf{0 . 0 0 0}$ \\
Apnea-to-bruxism index & $\mathbf{0 . 4 4}$ & $\mathbf{0 . 2 3}$ & 0.685 \\
Male gender & 0.66 & 1.62 & $\mathbf{0 . 0 4 1}$ \\
BMI & $\mathbf{0 . 5 3}$ & $\mathbf{0 . 2 6}$ & 0.472 \\
Age & 0.06 & 0.09 & \\
\hline
\end{tabular}

MAP, mean arterial pressure (mmHg); BMI, body mass index $\left(\mathrm{kg} / \mathrm{m}^{2}\right)$; Rc, regression coefficient; SEM, standard error of mean; statistically significant differences are marked as bold $(p<0.05)$.

This finding confirmed that a higher apnea-to-bruxism index and higher BMI were independent predictors of the increased average MAP during daytime in the studied group (Table 6). 
Table 6. Results of the estimation for the final model obtained on multivariate regression analysis. Model for average daytime MAP.

\begin{tabular}{lccc}
\hline \multirow{2}{*}{ Parameter } & \multicolumn{3}{c}{ Model for Average MAP (Daytime) } \\
\cline { 2 - 4 } & Rc & SEM of RC & $p$ \\
\hline Intercept & $\mathbf{6 9 . 0 1}$ & $\mathbf{5 . 1 4}$ & $\mathbf{0 . 0 0 0}$ \\
Apnea-to-bruxism index & $\mathbf{0 . 7 2}$ & $\mathbf{0 . 3 0}$ & $\mathbf{0 . 0 4 8}$ \\
Male gender & 1.28 & 1.76 & 0.468 \\
BMI & $\mathbf{0 . 5 9}$ & $\mathbf{0 . 2 8}$ & $\mathbf{0 . 0 3 6}$ \\
Age & 0.08 & 0.10 & 0.424 \\
\hline
\end{tabular}

MAP, mean arterial pressure (mmHg); BMI, body mass index $\left(\mathrm{kg} / \mathrm{m}^{2}\right)$; Rc, regression coefficient; SEM, standard error of mean; statistically significant differences are marked as bold $(p<0.05)$.

The regression analysis also indicated that a higher apnea-to-bruxism index, male gender, BEI $>4, \mathrm{BMI} \geq 25 \mathrm{~kg} / \mathrm{m}^{2}$, higher arousal index, and shorter TST were the independent risk factors for increased SBP variability during sleep period (Tables 7 and 8).

Table 7. Results of the estimation for the final model obtained on multivariate regression analysis. Model for nighttime SBP variability and anthropometric and polysomnographic characteristics.

\begin{tabular}{lccc}
\hline \multirow{2}{*}{ Parameter } & \multicolumn{3}{c}{ Model for SBP Variability (Nighttime) } \\
\cline { 2 - 4 } & Rc & SEM of RC & $p$ \\
\hline Intercept & $\mathbf{8 . 4 9}$ & $\mathbf{2 . 6 4}$ & $\mathbf{0 . 0 0 2}$ \\
Apnea-to-bruxism index & $\mathbf{0 . 3 3}$ & $\mathbf{0 . 1 1}$ & $\mathbf{0 . 0 4 0}$ \\
Male gender & $\mathbf{2 . 0 8}$ & $\mathbf{0 . 9 0}$ & $\mathbf{0 . 0 2 5}$ \\
BMI & -0.05 & 0.14 & 0.724 \\
Age & 0.04 & 0.05 & 0.434 \\
\hline
\end{tabular}

SBP, systolic blood pressure ( $\mathrm{mmHg})$; BMI, body mass index $\left(\mathrm{kg} / \mathrm{m}^{2}\right)$; Rc, regression coefficient; SEM, standard error of mean; statistically significant differences are marked as bold $(p<0.05)$.

Table 8. Results of the estimation for the final model obtained on multivariate regression analysis. Model for nighttime SBP variability and anthropometric, bruxism, and polysomnographic indices.

\begin{tabular}{lccc}
\hline \multirow{2}{*}{ Parameter } & \multicolumn{3}{c}{ Model for SBP Variability (Nighttime) } \\
\cline { 2 - 4 } & Rc & SEM of RC & $p$ \\
\hline Intercept & $\mathbf{1 1 . 4 8}$ & 3.76 & 0.004 \\
BEI $>4$ & 1.57 & 0.63 & 0.039 \\
BMI $\geq 25$ & 2.03 & 1.00 & 0.042 \\
Arousals & 0.18 & 0.09 & 0.044 \\
TST & $-\mathbf{0 . 0 1}$ & $\mathbf{0 . 0 1}$ & $\mathbf{0 . 0 2 6}$ \\
\hline
\end{tabular}

SBP, systolic blood pressure (mmHg); BEI, bruxism episode index ( $n /$ hour); BMI, body mass index ( $\left.\mathrm{kg} / \mathrm{m}^{2}\right)$; TST, total sleep time; Rc, regression coefficient; SEM, standard error of mean; statistically significant differences are marked as bold $(p<0.05)$.

\section{Discussion}

The results of the current study showed an association between SB intensity and blood pressure fluctuations. The results demonstrate that SB affects BP. The SBP variability at nighttime significantly increased in severe sleep bruxers as compared to those in the remaining study group. Blood pressure variability (BPV) reflects dynamic changes in BP caused by physical, emotional, and environmental factors. The clinical importance of BPV is broadly discussed as follows. BPV is considered a risk factor for cardiovascular events [29,30] and is associated with organ complications and cardiovascular mortality $[29,31]$ independent from mean $24 \mathrm{~h} \mathrm{BP}$ values. Therefore, cardiovascular reactivity and BP fluctuations promote an individual's risk of developing hypertension and can be considered to be equivalent to the traditional determinants of hypertension. Thus, the implications of the current findings involve serious risk of hypertension development in severe sleep bruxers 
without the actual diagnosis of hypertension. Moreover, regression analysis demonstrated that SB intensity was associated with increased SBP variability independently from anthropometric and polysomnographic parameters such as age, gender, BMI, and apnea events. Importantly, the current findings show a novel association between SB intensity and SBP variability independent from OSA. Lower minimal values of SBP at nighttime in severe sleep bruxers as compared to that in the remaining study group may be considered due to the sympathetic tone balance as argued previously [32,33].

This result highlights that little is known about the implications of SB for general health. Metabolic and hormonal disturbances related to the increased level of inflammatory and stress markers were found in young sleep bruxers in a prior study by our research team [34]. Several existing studies in the broader literature have examined clinical implications of SB on oral health and sleep-related disorders [9,23,35]. Seminal contributions to more comprehensive perspectives have been made by Huynh and Lavigne $[18,19,36]$ who investigated the role of autonomic cardiac activation in SB events. Finally, the authors demonstrated the co-occurrence of RMMA events, arousal, and respiratory events in patients with SB $[37,38]$.

The literature on the relationship between SB and hypertension confirmed our results. For instance, Nashed et al. [21] demonstrated that RMMA is associated with BP fluctuations during sleep. The methods were based on nocturnal beat-to-beat BP measurements. Although the study was well conducted and designed, the study group consisted of only 10 participants, whereas the control group had nine individuals. In a previous study by our research team, Martynowicz et al. [22] demonstrated that hypertension was an independent risk factor for SB. The lack of objective diagnosis of hypertension was the most important limitation of this previous study; hence, we designed the current study to diagnose hypertension and assess cardiovascular risk in bruxing, normotensive healthy patients by using ABPM.

It is also important to highlight the genetic basis of SB. The influence of neurotransmitters involved in sleep regulation, such as acetylcholine, noradrenaline, dopamine, and serotonin, on the genesis of SB has been insufficiently explored [39,40]. Wieckiewicz et al. [8] suggested that the genetic basis of primary bruxism is associated with the rs686 G variant of the dopamine receptor DRD1 encoding gene and the rs2770304 and rs6313 polymorphisms of the serotonin receptor HRT2A encoding gene. Their study also revealed a possible genetic association between SB and OSA, wherein a statistically significant correlation was observed within a group of HTR2A rs2770304 polymorphism TT homozygous cases. The serotonin receptor HRT2A has been linked previously with stress-induced response and anxiety-level mediation [41]. Serotoninergic mechanisms seem to play a crucial role in SB pathogenesis [8]. Therefore, the potential mechanism that causes decrease and variability in SBP in severe sleep bruxers could reflect the regulating effect of serotonin on the autonomic system activity [42]. Serotonin is also related to vasoconstriction and vasodilatation functions [43]. The results of the current study support this fact by showing that severe sleep bruxers had lower MAP in daytime. This finding is consistent with the assumption that nocturnal BP fluctuations cause dysregulations of the cardiovascular system.

A further relevant result is the correlation between BP measurements and SB events related to apnea. The study group included relatively young, normotensive subjects (mean age $33.96 \pm 11.22$ years) without severe comorbidities; therefore, the prevalence of OSA in this study group was considered to be a surprising finding. It is difficult to explain this result within the context of the normal weight of the study group (average BMI: $22.48 \pm 3.87 \mathrm{~kg} / \mathrm{m}^{2}$ ). Obesity is the major risk factor for OSA [44]. The remaining risk factors for OSA are well documented and include a family history of OSA, retrognathia, resistant hypertension, congestive heart failure, atrial fibrillation, stroke, and type 2 diabetes [45]. None of the participants from the study group met these criteria (risk factors). Thus, the high prevalence of OSA (21.54\%) in the study group without classical risk factors for OSA may suggest a secondary SB related to apneic events. This relationship was previously observed by Hosoya et al., Martynowicz et al., and Tan et al. All these authors 
have highlighted the protective features of SB against hypoxic event [46-48]. Moreover, the literature on SB has established sympathetic activity as the underlying reason for its consequences such as heart rate variability, hypertension, OSA, and hormonal and metabolic disturbances. A significant difference in the ABPM data between participants with an apnea-to-bruxism index $<1$ and those with an apnea-to-bruxism index $\geq 1$ supports this hypothesis. In the group with higher number of SB events related to apnea, increased BP values were found. Bruxism related to apnea events influenced both daytime and nighttime measurements of the increased BP values. In contrast, heart rate significantly decreased in the study group with an apnea-to-bruxism index $\geq 1$. A recent study of our research team provided some information related to the background of this issue and reported heart rate variability as an expression of sympathetic tone in SB and its consequences. [33] A significant decrease in heart rate was considered as a consequence of autonomic homeostasis in response to increased cardiac activity. Xu et al. demonstrated that in a study group with severe OSA, nocturnal and awake BP values were associated with the duration of hypoxia during sleep and BP fluctuations. In contrast to our present study design, BP was assessed in this study by measuring pulse transmit time (PTT) [49].

The broad implication of the present research is that SB in young patients with increased sympathetic tone favors the development of BP fluctuations [21] and subsequently hypertension. Sympathetic activity is modulated by an increased level of stress and anxiety, which are the major determinants of SB. Subsequently, contributory risk factors, such as age, gender, and weight gain, influence and determine OSA and hypertension progression, which are associated with SB. [47] The very basis of this positive feedback loop is sympathetic activity. The cause-and-effect relationship between increased sympathetic activity and blood pressure fluctuations in sleep bruxers suggest association between SB and increased risk of cardiovascular complications. Despite previous and current study are preliminary, authors suggest assessing cardiovascular and respiratory implications of SB in bruxing patients. Further studies should aim to provide more information about this health prevention concern.

The present study had some limitations. First, the results are specific to young, healthy, normotensive adult subjects, and we did not diagnose elderly patients or children. It could also be argued whether BP fluctuations in sleep bruxers could affect co-existing cardiovascular conditions. Further studies on comparison between SB patients with cardiovascular diseases and controls are needed to determine whether SB affects BP alterations and worsens CVD. Future studies should aim to replicate the results in vulnerable individuals. Second, there was a lack of parity in the study group, and women constituted $69.23 \%$ of the study participants. This apparent limitation could influence our findings, as has been revealed in a recent study by Smardz et al. [50] where age and gender were found to influence SB. Last but not least, the participants underwent one-night PSG without an adaptation due to the presence of technical issues.

To summarize, the results of our present study shed new light on SB comorbidity, especially BP fluctuations and warrants a change in the clinical approach to patients with SB.

\section{Conclusions}

1. SB intensity is associated with increased SBP variability at nighttime (sleep period);

2. Coexisting apnea is considered to be the main determinant that influences BP in normotensive sleep bruxers;

3. A future approach should consider the potential risk of hypertension or abnormalities in BP regulation and other cardiovascular risk factors in sleep bruxers more carefully as potentially significant implications for health. 
Author Contributions: Validation, P.G.; formal analysis, P.G. and R.P.; investigation, H.M., A.W. and J.S.; resources, M.M.-Z.; data curation, M.M.-Z., K.G. and A.W.; writing-original draft preparation, M.M.-Z. and M.W.; writing—review and editing, H.M., P.G., M.W. and R.P.; visualization, M.W.; supervision, G.M. All authors have read and agreed to the published version of the manuscript.

Funding: This research received no external funding.

Institutional Review Board Statement: The study was approved by the local Ethics Committee (no. KB-195/2017) and was conducted by adopting the principles of the Declaration of Helsinki. This clinical trial was also registered in Clinical Trials Database (www.ClinicalTrials.gov; access date: 20 March 2021): identifier NCT03083405, WMU1/2017.

Informed Consent Statement: Informed consent was obtained from all subjects involved in the study.

Data Availability Statement: The data presented in this study are available on request from the corresponding author. The data are not publicly available due to European Union restrictions.

Conflicts of Interest: The authors declare no conflict of interest.

\section{References}

1. Calhoun, D.A.; Harding, S.M. Sleep and hypertension. Chest 2010, 138, 434-443. [CrossRef] [PubMed]

2. $\quad$ Staessen, J.A.; Yang, W.Y.; Melgarejo, J.D.; Thijs, L.; Zhang, Z.Y.; Boggia, J.; Wei, F.F.; Hansen, T.W.; Asayama, K.; Ohkubo, T.; et al. Association of Office and Ambulatory Blood Pressure with Mortality and Cardiovascular Outcomes. JAMA J. Am. Med. Assoc. 2019, 322, 409-420. [CrossRef]

3. Di Raimondo, D.; Musiari, G.; Pinto, A. Nocturnal blood pressure patterns and cardiac damage: There is still much to learn. Hypertens. Res. 2020, 43, 246-248. [CrossRef]

4. Gangwisch, J.E. A review of evidence for the link between sleep duration and hypertension. Am. J. Hypertens. 2014, 27, 1235-1242. [CrossRef] [PubMed]

5. Jarrin, D.C.; Alvaro, P.K.; Bouchard, M.A.; Jarrin, S.D.; Drake, C.L.; Morin, C.M. Insomnia and hypertension: A systematic review. Sleep Med. Rev. 2018, 41, 3-38. [CrossRef]

6. Van Den Eeden, S.K.; Albers, K.B.; Davidson, J.E.; Kushida, C.A.; Leimpeter, A.D.; Nelson, L.M.; Popat, R.; Tanner, C.M.; Bibeau, K.; Quesenberry, C.P. Risk of cardiovascular disease associated with a restless legs syndrome diagnosis in a retrospective cohort study from Kaiser Permanente Northern California. Sleep 2015, 38, 1009-1015. [CrossRef] [PubMed]

7. Hou, H.; Zhao, Y.; Yu, W.; Dong, H.; Xue, X.; Ding, J.; Xing, W.; Wang, W. Association of obstructive sleep apnea with hypertension: A systematic review and meta-analysis. J. Glob. Health 2018, 8, 1-10. [CrossRef] [PubMed]

8. Wieckiewicz, M.; Bogunia-Kubik, K.; Mazur, G.; Danel, D.; Smardz, J.; Wojakowska, A.; Poreba, R.; Dratwa, M.; ChaszczewskaMarkowska, M.; Winocur, E.; et al. Genetic basis of sleep bruxism and sleep apnea-Response to a medical puzzle. Sci. Rep. 2020, 10, 1-14. [CrossRef]

9. Mayer, P.; Heinzer, R.; Lavigne, G. Sleep bruxism in respiratory medicine practice. Chest 2016, 149, 262-271. [CrossRef] [PubMed]

10. Manfredini, D.; Winocur, E.; Guarda-Nardini, L.; Paesani, D.; Lobbezoo, F. Epidemiology of Bruxism in Adults: A Systematic Review of the Literature. J. Orofac. Pain 2013, 27, 99-110. [CrossRef]

11. Sateia, M.J. International Classification of Sleep Disorders, 3rd ed.; American Academy of Sleep Medicine: Darien, IL, USA, 2014.

12. Van Ryswyk, E.; Mukherjee, S.; Chai-Coetzer, C.L.; Vakulin, A.; Mcevoy, R.D. Sleep disorders, including sleep apnea and hypertension. Am. J. Hypertens. 2018, 31, 857-864. [CrossRef]

13. Javier Nieto, F.; Young, T.B.; Lind, B.K.; Shahar, E.; Samet, J.M.; Redline, S.; D'Agostino, R.B.; Newman, A.B.; Lebowitz, M.D.; Pickering, T.G. Association of sleep-disordered breathing sleep apnea, and hypertension in a large community-based study. J. Am. Med. Assoc. 2000, 283, 1829-1836. [CrossRef]

14. Peppard, P.E.; Young, T.; Palta, M.; Skatrud, J. Prospective Study of the Association between Sleep-Disordered Breathing and Hypertension. N. Engl. J. Med. 2000, 342, 1378-1384. [CrossRef]

15. Marin, J.M.; Agusti, A.; Villar, I.; Forner, M.; Nieto, D.; Carrizo, S.J.; Barbé, F.; Vicente, E.; Wei, Y.; Javier Nieto, F.; et al. Association between treated and untreated obstructive sleep apnea and risk of hypertension. JAMA J. Am. Med. Assoc. 2012, 307, 2169-2176. [CrossRef] [PubMed]

16. Mohsenin, V. Obstructive Sleep Apnea and Hypertension: A Critical Review. Curr. Hypertens. Rep. 2014, 16, 482. [CrossRef]

17. Salman, L.A.; Shulman, R.; Cohen, J.B. Obstructive Sleep Apnea, Hypertension, and Cardiovascular Risk: Epidemiology, Pathophysiology, and Management. Curr. Cardiol. Rep. 2020, 22, 6. [CrossRef]

18. Huynh, N.; Kato, T.; Rompré, P.H.; Okura, K.; Saber, M.; Lanfranchi, P.A.; Montplaisir, J.Y.; Lavigne, G.J. Sleep bruxism is associated to micro-arousals and an increase in cardiac sympathetic activity. J. Sleep Res. 2006, 15, 339-346. [CrossRef]

19. Lavigne, G.J.; Huynh, N.; Kato, T.; Okura, K.; Adachi, K.; Yao, D.; Sessle, B. Genesis of sleep bruxism: Motor and autonomiccardiac interactions. Arch. Oral Biol. 2007, 52, 381-384. [CrossRef] [PubMed]

20. Fisher, J.P.; Paton, J.F.R. The sympathetic nervous system and blood pressure in humans: Implications for hypertension. J. Hum. Hypertens. 2012, 26, 463-475. [CrossRef] [PubMed] 
21. Nashed, A.; Lanfranchi, P.; Rompré, P.; Carra, M.C.; Mayer, P.; Colombo, R.; Huynh, N.; Lavigne, G. Sleep Bruxism Is Associated with a Rise in Arterial Blood Pressure. Sleep 2012, 35, 529-536. [CrossRef]

22. Martynowicz, H.; Dymczyk, P.; Dominiak, M.; Kazubowska, K.; Skomro, R.; Poreba, R.; Gac, P.; Wojakowska, A.; Mazur, G.; Wieckiewicz, M. Evaluation of Intensity of Sleep Bruxism in Arterial Hypertension. J. Clin. Med. 2018, 7, 327. [CrossRef]

23. Carra, M.C.; Huynh, N.; Fleury, B.; Lavigne, G. Overview on Sleep Bruxism for Sleep Medicine Clinicians. Sleep Med. Clin. 2015, 10, 375-384. [CrossRef]

24. Lobbezoo, F.; Ahlberg, J.; Raphael, K.G.; Wetselaar, P.; Glaros, A.G.; Kato, T.; Santiago, V.; Winocur, E.; De Laat, A.; De Leeuw, R.; et al. International consensus on the assessment of bruxism: Report of a work in progress. J. Oral Rehabil. 2018, 45, 837-844. [CrossRef]

25. Berry, R.B.; Brooks, R.; Gamaldo, C.E.; Harding, S.M.; Marcus, C.; Vaughn, B.V. The AASM manual for the scoring of sleep and associated events. Rules, Terminology and Technical Specifications, Darien, Illinois. Am. Acad. Sleep Med. 2012, 176, 2012.

26. Saito, M.; Yamaguchi, T.; Mikami, S.; Watanabe, K.; Gotouda, A.; Okada, K.; Hishikawa, R.; Shibuya, E.; Lavigne, G. Temporal association between sleep apnea-hypopnea and sleep bruxism events. J. Sleep Res. 2013. [CrossRef] [PubMed]

27. Williams, B.; Mancia, G.; Spiering, W.; Rosei, E.A.; Azizi, M.; Burnier, M.; Clement, D.L.; Coca, A.; De Simone, G.; Dominiczak, A.; et al. 2018 ESC/ESH Guidelines for the management of arterial hypertension. Kardiol. Pol. 2019, 77, 71-159. [CrossRef] [PubMed]

28. O’Brien, E.; Parati, G.; Stergiou, G.; Asmar, R.; Beilin, L.; Bilo, G.; Clement, D.; De La Sierra, A.; De Leeuw, P.; Dolan, E.; et al. European society of hypertension position paper on ambulatory blood pressure monitoring. J. Hypertens. 2013, 31, 1731-1768. [CrossRef] [PubMed]

29. Parati, G.; Stergiou, G.S.; Dolan, E.; Bilo, G. Blood pressure variability: Clinical relevance and application. J. Clin. Hypertens. 2018, 20, 1133-1137. [CrossRef] [PubMed]

30. Parati, G.; Ochoa, J.E.; Lombardi, C.; Bilo, G. Assessment and management of blood-pressure variability. Nat. Rev. Cardiol. 2013, 10, 143-155. [CrossRef]

31. Stevens, S.L.; Wood, S.; Koshiaris, C.; Law, K.; Glasziou, P.; Stevens, R.J.; McManus, R.J. Blood pressure variability and cardiovascular disease: Systematic review and meta-analysis. BMJ 2016, 354, i4098. [CrossRef]

32. Marthol, H.; Reich, S.; Jacke, J.; Lechner, K.H.; Wichmann, M.; Hilz, M.J. Enhanced sympathetic cardiac modulation in bruxism patients. Clin. Auton. Res. 2006, 16, 276-280. [CrossRef]

33. Michalek-Zrabkowska, M.; Wieckiewicz, M.; Macek, P.; Gac, P.; Smardz, J.; Wojakowska, A.; Poreba, R.; Mazur, G.; Martynowicz, H. The Relationship between Simple Snoring and Sleep Bruxism: A Polysomnographic Study. Int. J. Environ. Res. Public Health 2020, 17, 8960. [CrossRef]

34. Michalek-Zrabkowska, M.; Wieckiewicz, M.; Smardz, J.; Gac, P.; Poreba, R.; Wojakowska, A.; Mazur, G.; Martynowicz, H. Determination of Inflammatory Markers, Hormonal Disturbances, and Sleepiness Associated with Sleep Bruxism Among Adults. Nat. Sci. Sleep 2020, 12, 969. [CrossRef] [PubMed]

35. Beddis, H.; Pemberton, M.; Davies, S. Sleep bruxism: An overview for clinicians. Br. Dent. J. 2018, 225, 497-501. [CrossRef]

36. Lavigne, G.J.; Kato, T.; Kolta, A.; Sessle, B.J. Neurobiologicalmechanisms involved in sleep bruxism. Crit. Rev. Oral Biol. Med. 2003, 14, 30-46. [CrossRef] [PubMed]

37. Huynh, N.; Lavigne, G.J.; Lanfranchi, P.A.; Montplaisir, J.Y.; De Champlain, J. The effect of 2 sympatholytic medicationsPropranolol and clonidine-On sleep bruxism: Experimental randomized controlled studies. Sleep 2006, 29, 307-316. [CrossRef]

38. Kato, T.; Montplaisir, J.Y.; Guitard, F.; Sessle, B.J.; Lund, J.P.; Lavigne, G.J. Evidence that experimentally induced sleep bruxism is a consequence of transient arousal. J. Dent. Res. 2003, 82, 284-288. [CrossRef]

39. Lavigne, G.J.; Khoury, S.; Abe, S.; Yamaguchi, T.; Raphael, K. Bruxism physiology and pathology: An overview for clinicians. J. Oral Rehabil. 2008, 35, 476-494. [CrossRef]

40. Saper, C.B.; Scammell, T.E.; Lu, J. Hypothalamic regulation of sleep and circadian rhythms. Nature 2005, 437, 1257-1263. [CrossRef] [PubMed]

41. Bayar, G.R.; Tutuncu, R.; Acikel, C. Psychopathological profile of patients with different forms of bruxism. Clin. Oral Investig. 2012, 16, 305-311. [CrossRef]

42. Frazer, A.; Hensler, J.G. Serotonin Involvement in Physiological Function and Behavior. In Basic Neurochemistry: Molecular, Cellular and Medical Aspects, 6th ed.; Siegel, G.J., Agranoff, B.W., Albers, R.W., Fisher, S.K., Uhler, M., Eds.; Lippincott-Raven: Philadelphia, PA, USA, 1999.

43. Watts, S.W. Serotonin and sensory nerves: Meeting in the cardiovascular system. Vascul. Pharmacol. 2014, 63, 1-3. [CrossRef]

44. Hamilton, G.S.; Joosten, S.A. Obstructive sleep apnoea and obesity. Aust. Fam. Physician 2017, 46, 460-463. [PubMed]

45. Patel, S.R. Obstructive sleep apnea. Ann. Intern. Med. 2019, 171, ITC81-ITC96. [CrossRef]

46. Hosoya, H.; Kitaura, H.; Hashimoto, T.; Ito, M.; Kinbara, M.; Deguchi, T.; Irokawa, T.; Ohisa, N.; Ogawa, H.; Takano-Yamamoto, T. Relationship between sleep bruxism and sleep respiratory events in patients with obstructive sleep apnea syndrome. Sleep Breath. 2014, 18, 837-844. [CrossRef] [PubMed]

47. Martynowicz, H.; Gac, P.; Brzecka, A.; Poreba, R.; Wojakowska, A.; Mazur, G.; Smardz, J.; Wieckiewicz, M. The Relationship between Sleep Bruxism and Obstructive Sleep Apnea Based on Polysomnographic Findings. J. Clin. Med. 2019, 8, 1653. [CrossRef] [PubMed] 
48. Tan, M.; Yap, A.; Chua, A.; Wong, J.; Parot, M.; Tan, K. Prevalence of Sleep Bruxism and Its Association with Obstructive Sleep Apnea in Adult Patients: A Retrospective Polysomnographic Investigation. J. Oral Facial Pain Headache 2019, 33, $269-277$. [CrossRef] [PubMed]

49. Xu, J.; Ding, N.; Zhang, X.; Wang, N.; Sun, B.; Zhang, R.; Xie, X.; Wan, Z.; Gu, Y.; Zhang, S.; et al. Nocturnal blood pressure fluctuation and associated influential factors in severe obstructive sleep apnea patients with hypertension. Sleep Breath. 2018, 22, 1045-1052. [CrossRef]

50. Smardz, J.; Wieckiewicz, M.; Gac, P.; Poreba, R.; Wojakowska, A.; Mazur, G.; Martynowicz, H. Influence of age and gender on sleep bruxism and snoring in non-apneic snoring patients: A polysomnographic study. J. Sleep Res. 2020. [CrossRef] 